\title{
Note on a problem of Ruzsa
}

\author{
by
}

\section{Norbert HEGyvári (Budapest)}

1. Introduction. Let $B=\left\{1 \leq b_{1}<b_{2}<\ldots\right\}$ be an infinite sequence of integers. For any integer $n$ we define the counting function of $B$ up to $n$ to be the number of elements of $B$ not exceeding $n$; we denote it by $B(n)$. The lower asymptotic density $\underline{d} B$ and the upper asymptotic density $\bar{d} B$ are defined by

$$
\underline{d} B=\liminf _{n \rightarrow \infty} B(n) / n, \quad \bar{d} B=\limsup _{n \rightarrow \infty} B(n) / n .
$$

If $\underline{d} B=\bar{d} B$, we say that $B$ has asymptotic density $d B$, given by the common value.

In [3] I. Z. Ruzsa proved that if $A=\left\{1 \leq a_{1}<a_{2}<\ldots\right\}$ is an infinite sequence of integers and if $a_{n+1} \leq 2 a_{n}$ for all but at most finitely many values of $n$, then $P(A)$ has an asymptotic density, where $P(A)$ is the set of all sums of the form $\sum \varepsilon_{i} a_{i}, \varepsilon_{i}=0$ or 1 . Ruzsa conjectured that for every pair of numbers $0 \leq \alpha \leq \beta \leq 1$ there exists $A=\left\{1 \leq a_{1}<a_{2}<\ldots\right\}$ for which $\underline{d}(P(A))=\alpha$ and $\bar{d}(P(A))=\beta$. He also mentioned that an easy argument shows the case $\beta=1$.

In this paper we prove Ruzsa's conjecture:

TheOrem. Let $0 \leq \alpha \leq \beta \leq 1$. Then there exists an $A=\left\{a_{1}<a_{2}<\ldots\right\}$ such that

$$
\underline{d}(P(A))=\alpha \quad \text { and } \quad \bar{d}(P(A))=\beta .
$$

The finite version of this question may be the following: for which $t$ is it possible to find a sequence $a_{1}<\ldots<a_{n}$ so that there are exactly $t$ distinct integers of the form $\sum_{i=1}^{n} \varepsilon_{i} a_{i}, \varepsilon_{i}=0$ or 1. It was raised in [1] and solved in $[2]$.

Acknowledgements. I would like to thank the referee for his helpful comments and suggestions.

Supported by CNRS Laboratoire de Mathématiques Discrètes, Marseille. 
2. The construction. If $\alpha=\beta \neq 0$ then it is easy to see that $d(P(A))$ $=\alpha$ for $A=\left\{\left[2^{n} / \alpha\right] \mid n \in \mathbb{N}\right\}$; if $\alpha=\beta=0$ then clearly $d(P(A))=0$ for $A=\left\{2^{2^{n}} \mid n \in \mathbb{N}\right\}$. So assume that $0 \leq \alpha<\beta \leq 1$.

We use the following notation: $A=\left\{a_{1}<a_{2}<\ldots\right\}, A_{n}=\left\{a_{1}<\right.$ $\left.\ldots<a_{n}\right\} ; s_{n}=\sum_{i=1}^{n} a_{i} ; \varrho_{n}=\left|P\left(A_{n}\right)\right| / s_{n} ; p_{n}(x)=\left|P\left(A_{n}\right) \cap[1, x]\right| ; \tau_{n}=$ $p_{n-1}\left(a_{n}\right) / a_{n}$.

Let $A=\bigcup_{i=0}^{\infty} \mathcal{B}_{i}$, where the blocks $\mathcal{B}_{i}$ will be determined by an iterative process.

First let us define the block $\mathcal{B}_{0}$. Let $k_{0}=\max \{[18 \beta / \alpha]+8,[18 \beta]+$ $2,2 /(\beta-\alpha)\}$ and let $\mathcal{B}_{0}=\left\{a_{1}<\ldots<a_{k_{0}}\right\}$, where

$$
a_{i}= \begin{cases}2^{i} & \text { if } 0 \leq i \leq k_{0}-1 \\ \min \left\{x \mid\left(2^{k_{0}+1}-2\right) /\left(x+2^{k_{0}}\right) \leq \beta\right\} & \text { for } i=k_{0} .\end{cases}
$$

Thus $P\left(A_{k_{0}}\right)=\left[1,2^{k_{0}}-1\right] \cup\left[x+1, x+2^{k_{0}}-1\right]$ and so

$$
\varrho_{k_{0}}=\left(2^{k_{0}+1}-1\right) /\left(x+2^{k_{0}}\right) \leq \beta
$$

and an easy calculation shows that $\varrho_{k_{0}} \geq \beta-1 / k_{0}$.

Assume now that the blocks $\mathcal{B}_{1}, \ldots, \mathcal{B}_{j-1}$ have been defined such that for each $1 \leq m \leq j-1$,

$$
\mathcal{B}_{m}=\left\{a_{N_{1}^{(m)}}<\ldots<a_{N_{2}^{(m)}}<\ldots<a_{N_{3}^{(m)}}\right\}
$$

where $s_{N_{3}^{(m-1)}}<a_{N_{1}^{(m)}}$ with $N_{1}^{(m)}=N_{3}^{(m-1)}+1$ and $a_{k_{0}}<a_{N_{1}^{(1)}}$ with $N_{1}(1)=k_{0}+1$. Furthermore, if $k:=k_{0}+m$, then for every $m, 1 \leq m \leq j$, the following properties are true:

$$
\begin{gathered}
\alpha \leq \tau_{N_{1}^{(k)}} \leq \alpha+1 / k, \\
\varrho_{N_{2}^{(k)}}>\beta / 3, \\
\beta-1 / k \leq \varrho_{N_{3}^{(k)}} \leq \beta .
\end{gathered}
$$

Our task is to define blocks $\mathcal{B}_{j}, \mathcal{B}_{j+1}, \ldots$ so that the properties (2)-(4) remain valid for $k=k_{0}+m, m \geq j$ as well. We verify these parallel with the construction.

In the last section we prove that for $x>s_{k_{0}}$,

$$
\alpha \leq|P(A) \cap[1, x]| / x \leq \beta .
$$

Now we note that $(2),(4)$ and (5) imply

$$
\underline{d}(P(A))=\alpha \quad \text { and } \quad \bar{d}(P(A))=\beta .
$$

Indeed, by (2) and (4) we have

$$
\lim _{k \rightarrow \infty}\left|P\left(A_{N_{1}^{(k)}}\right)\right| / a_{N_{1}^{(k)}}=\alpha, \quad \lim _{k \rightarrow \infty}\left|P\left(A_{N_{3}^{(k)}}\right)\right| / s_{N_{3}^{(k)}}=\beta,
$$

and by (5) we get (1). 
3. Proof of the Theorem. Now we prepare the block $\mathcal{B}_{k}$.

We use the abbreviations $N_{i}=N_{i}^{(k)}$ for $i=1,2,3$ and let $N_{0}=N_{3}^{(k-1)}$. In the first step we make the sequence less dense. Let

$$
a_{N_{1}}=\max \left\{y\left|y>s_{N_{0}},\right| P\left(A_{N_{0}}\right) \mid / y \geq \alpha\right\} .
$$

Since $1 / 2^{k_{0}}<1 / k_{0}<\beta-\alpha, y$ exists.

Since $N_{1}>k_{0}+j$, this definition implies

$$
\begin{aligned}
0 \leq \tau_{N_{1}}-\alpha & =\left|P\left(A_{N_{0}}\right)\right| / a_{N_{1}}-\alpha<\left|P\left(A_{N_{0}}\right)\right| / a_{N_{1}}-\left|P\left(A_{N_{0}}\right)\right| /\left(a_{N_{1}}+1\right) \\
& =\left|P\left(A_{N_{0}}\right)\right| /\left\{a_{N_{1}}\left(a_{N_{1}}+1\right)<\alpha / a_{N_{1}}<1 / N_{1}<1 / k,\right.
\end{aligned}
$$

$1 \leq k_{0}<k$, showing (2).

In the next step we do two things: we "stabilize" the density of our sequence and then we make it more dense up to $\beta / 3$.

Let $M=a_{N_{1}}$. Let

$$
a_{N_{1}+i}=(i+1) a_{N_{1}}
$$

for $i=1, \ldots, M$ and if $t:=\left[a_{N_{1}} / s_{N_{0}}\right] \geq 2$ then let

$$
a_{N_{1}+M+i}=(M+i+1) a_{N_{1}}+s_{N_{0}}
$$

for $i=1, \ldots, t-1$. The elements defined in (7) stabilize the density and the ones defined in (8) will make the density close to $\beta / 3$, which we now show.

Let $N_{2}=N_{1}+M+t-1$. Then $\varrho_{N_{2}} \geq \beta / 3$. Indeed, if $t<2$ then by (4), (7) and since $k>k_{0}>3 / \beta+1$, we have

$$
\varrho_{N_{2}}>\varrho_{N_{1}-1} / 2=\varrho_{N_{0}} / 2>(\beta-1 /(k-1)) / 2>\beta / 3 .
$$

Let now $t \geq 2$ and let $M+t \leq j \leq\left(\begin{array}{c}M+1 \\ 2\end{array}\right)$. Clearly $P\left(A_{t}\right)=P\left(A_{t-1}\right) \cup$ $\left\{a_{t}+P\left(A_{t-1}\right)\right\}$ for every $t \in \mathbb{N}$. Since $a_{N_{1}}>s_{N_{0}}$ and by $(7)$ we see that $w \in P\left(A_{N_{2}}\right) \cap\left[j a_{N_{1}},(j+1) a_{N_{1}}\right]$ if and only if there exist $v \in P\left(A_{N_{0}}\right)$ and $z, 1 \leq z \leq\left(\begin{array}{c}M+1 \\ 2\end{array}\right)$, so that $w=z a_{N_{1}}+v$. So we have

$$
\left|P\left(A_{N_{2}}\right) \cap\left[j a_{N_{1}},(j+1) a_{N_{1}}\right)\right|=t\left|P\left(A_{N_{0}}\right)\right|
$$

and by (8),

$$
s_{N_{2}} \leq\left(\begin{array}{c}
M+t+2 \\
2
\end{array}\right) a_{N_{1}} .
$$

Furthermore, if $\left(\begin{array}{c}M+1 \\ 2\end{array}\right) \leq j \leq\left(\begin{array}{c}M+t+1 \\ 2\end{array}\right)$ then it is easy to check that

(11) $P\left(A_{N_{2}}\right) \cap\left[j a_{N_{1}},(j+1) a_{N_{1}}\right]=j a_{N_{1}}+\left\{u s_{N_{0}}+P\left(A_{N_{0}}\right) \mid 0 \leq u \leq t\right\}$.

Hence

$$
2 \leq t \leq a_{N_{1}} / s_{N_{0}}=\left\{\left|P\left(A_{N_{0}}\right)\right| / s_{N_{0}}\right\}\left\{a_{N_{1}} /\left|P\left(A_{N_{0}}\right)\right|\right\}=\varrho_{N_{0}} / \tau_{N_{1}}<\beta / \alpha
$$

so we get

$$
\beta>2 \alpha .
$$


Since $M>a_{N_{1}}>N_{1}>k_{0}$ by (9), (10) and (12) we get

(13) $\varrho_{N_{2}}$

$$
\begin{aligned}
& =\left|P\left(A_{N_{2}}\right)\right| / s_{N_{2}} \geq\left\{\left(\begin{array}{c}
M+1 \\
2
\end{array}\right)-(M+t)\right\} t\left|P\left(A_{N_{0}}\right)\right| /\left(\begin{array}{c}
M+t+2 \\
2
\end{array}\right) a_{N_{1}} \\
& \geq \frac{\left(\begin{array}{c}
M+1 \\
2
\end{array}\right)-(M+t)}{\left(\begin{array}{c}
M+t+2 \\
2
\end{array}\right)}\left(a_{N_{1}} / s_{N_{0}}-1\right)\left|P\left(A_{N_{0}}\right)\right| / a_{N_{1}} \\
& \geq\left((1-2 t / M)^{2}-2 / M\right)\left(\left|P\left(A_{N_{0}}\right)\right| / s_{N_{0}}-\left|P\left(A_{N_{0}}\right)\right| / a_{N_{1}}\right) \\
& \geq\left\{\left(1-2 \beta /\left(\alpha k_{0}\right)\right)^{2}-2 / k_{0}\right\}\left\{\beta-1 / k_{0}-\alpha\right\} \\
& \geq\left(1-4 \beta /\left(\alpha k_{0}\right)-2 / k_{0}\right)\left(\beta / 2-1 / k_{0}\right) \geq \beta / 3 .
\end{aligned}
$$

For the last inequality we use $k_{0}>16 \beta / \alpha+8$ and thus $1-4 \beta /\left(\alpha k_{0}\right)-2 / k_{0}>$ $3 / 4$; furthermore, $k_{0}>18 / \beta$ and thus $\beta / 2-1 / k_{0}>4 \beta / 9$. This proves (3).

In the next step we achieve that the sequence will be more dense, satisfying (4). Let $v=s_{N_{2}}$. Let

$$
a_{N_{2}+i}=2^{i} s_{N_{2}}
$$

for $i=1, \ldots, v$. This definition implies that $a_{N_{2}+i}>s_{N_{2}+i-1}$ and so

$$
\varrho_{N_{2}+v}=\varrho_{N_{2}} .
$$

Write for short $N=N_{2}+v ; W=s_{N}$ and $Y=\left[W \min \left\{1 / 2, \beta / \varrho_{N}-1\right\}\right]$ and $L=s_{N_{2}}^{2}$. Let now

$$
K_{W}(z)=\left|P\left(A_{N}\right) \cap\left(P\left(A_{N}\right)+W-Y+z\right)\right|
$$

for $0 \leq z \leq L$.

Lemma. There exists a $z^{*} \in[0, L]$ such that

$$
K_{W}\left(z^{*}\right) \leq Y\left(\varrho_{N}^{2}+3 / s_{N_{2}}\right) .
$$

Proof. Let $K_{W}=\sum_{z=0}^{L} K_{W}(z) / L$. By (14) we have

$$
\left|P\left(A_{N}\right) \cap[t, t+L]\right|<\varrho_{N} L+s_{N_{2}}=\left(\varrho+1 / s_{N_{2}}\right) L
$$

for $t=0, \ldots, s_{N}-L$ and

$$
\left|P\left(A_{N}\right) \cap[W-Y, W]\right|<Y\left(\varrho_{N}+1 / s_{N_{2}}\right) .
$$

Write

$$
\mathcal{H}=P\left(A_{N}\right) \cap[W-Y, W], \quad \mathcal{L}_{z}=W-Y+z+P\left(A_{N}\right) .
$$

Then by (17) and (18) we have

$$
K_{W} \leq \sum_{z=0}^{L} \sum_{u \in \mathcal{H} \cap \mathcal{L}_{z}} 1 \leq\left(\varrho_{N}+1 / s_{N_{2}}\right)^{2} \cdot L \cdot Y / L<\left(\varrho_{N}^{2}+3 / s_{N_{2}}\right) Y .
$$


This implies that

$$
K_{W}\left(z^{*}\right):=\min _{0 \leq z \leq L} K_{W}(z) \leq K_{W}<\left(\varrho_{N}^{2}+3 / s_{N_{2}}\right) Y
$$

which proves the lemma.

Let

$$
a_{N+1}=W-Y+z^{*} .
$$

Now we deduce a lower estimate for $\varrho_{N+1}$. By the Lemma we get

$$
\begin{aligned}
\varrho_{N+1}= & \frac{2 W \varrho_{N}-K_{W}\left(z^{*}\right)}{2 W-Y+z^{*}} \geq \frac{2 W \varrho_{N}-\left(\varrho_{N}^{2}+3 / s_{N_{2}}\right) Y}{2 W-Y+z^{*}} \\
\geq & \varrho_{N}+Y \varrho_{N}\left(1-\varrho_{N}\right) /\left(2 W-Y+z^{*}\right) \\
& -z^{*} /(2 W-Y)-3 Y /\left(s_{N_{2}}(2 W-Y)\right) .
\end{aligned}
$$

Let

$$
\omega_{N}=z^{*} /(2 W-Y)-3 Y /\left(s_{N_{2}}(2 W-Y)\right) .
$$

Clearly

$$
\lim _{N \rightarrow \infty} \omega_{N}=0 .
$$

by $(20)$

First case: $Y=\left[W \min \left\{1 / 2, \beta / \varrho_{N}-1\right\}\right]=\left[W\left(\beta / \varrho_{N}-1\right)\right]$. Then

(21) $\varrho_{N+1}$

$\geq \varrho_{N}+W\left(\beta / \varrho_{N}-1\right) \varrho_{N}\left(1-\varrho_{N}\right) /\left\{2 W-W\left(\beta / \varrho_{N}-1\right)+z^{*}\right\}-\left(\omega_{N}+1 / W\right)$

$=\varrho_{N}+\left(\beta-\varrho_{N}\right) \varrho_{N}\left(1-\varrho_{N}\right) /\left\{\left(3 \varrho_{N}-\beta\right)+\varrho_{N} z^{*} / W\right\}-\left(\omega_{N}+1 / W\right)$.

Since $\beta / 3<\varrho_{N}<\beta \leq 1$ the relation $\left(\beta-\varrho_{N}\right) \varrho_{N}\left(1-\varrho_{N}\right) /\left\{\left(3 \varrho_{N}-\beta\right)+\right.$ $\left.\varrho_{N} z^{*} / W\right\}>0$ holds. This implies that if $W$ (and so $N$ ) is large enough we have

$$
\varrho_{N+1}>\varrho_{N}
$$

Repeating the previous process we define by (14) and (19) the sequence $a_{N+2}, a_{N+3}, \ldots$ More precisely, let $N^{(1)}=N+1$ and define $a_{N^{(1)}}$ by (14) and $a_{N^{(2)}}$ by (19) and if $N^{(1)}, N^{(2)}, \ldots, N^{(2 r)}$ have been defined then let $N^{(2 r+1)}=N^{(2 r)}+1$ and define $a_{N^{(2 r+1)}}$ by (14) and $a_{N^{(2 r+2)}}$ by (19). Then (22) yields that $\beta / \varrho_{N+1}-1<\beta / \varrho_{N}$ so at each step of the iterative process described above we always fall in the first case. Since $\varrho_{N^{(i)}} \leq \beta$ and also by (22) we conclude that $\lim _{i \rightarrow \infty} \varrho_{N^{(i)}}=\lambda$ exists and clearly $\lambda \leq \beta$. Thus by (21) we get

$$
\lambda \geq \lambda+(\beta-\lambda) \lambda(1-\lambda) /(3 \lambda-\beta),
$$

which implies $\lambda=\beta$. Hence there is an $i \in \mathbb{N}$ such that $\beta-1 / k \leq \varrho_{N^{(i)}} \leq \beta$. So choosing $N_{3}=N^{(i)}$ we get (4). 
Second case: $Y=[W / 2]$. Then by $(20)$ we have

$$
\varrho_{N+1} \geq \varrho_{N}+(W / 2) \varrho_{N}\left(1-\varrho_{N}\right) /(2 W-W / 2)+\omega_{N}^{\prime},
$$

where $\lim \omega_{N}^{\prime}=0$. This implies that $\varrho_{N+1} \geq \varrho_{N}$ if $W$ (and so $N$ ) is large enough. Repeating the previous processes which are defined by (14) and (19) we see that $\lim _{i \rightarrow \infty} \varrho_{N^{(i)}}=\mu$ exists. By (23) we conclude that $\mu \geq 1$ thus there is an $i \in \mathbb{N}$ for which $\min \left\{1 / 2, \beta / \varrho_{N^{(i)}}-1\right\}=\beta / \varrho_{N^{(i)}}-1$ so we can use case 1 .

4. Proof of property (5). We divide the interval $\left[s_{k_{0}}, \infty\right)$ into the union

$$
\left[s_{k_{0}}, \infty\right)=\bigcup_{k \geq k_{0}}\left[s_{N_{3}^{(k-1)}}, s_{N_{3}^{(k)}}\right) .
$$

We now prove by induction on $k$ that if

$$
s_{N_{3}^{(k-1)}} \leq x<s_{N_{3}^{(k)}}
$$

for some $k$ then (5) is true.

First, note that if we choose $a_{N_{1}^{(k)}}$ at each step $\left(a_{N_{1}^{(k)}}\right.$ is the initial element of the block $\mathcal{B}_{k}$ ) then since $a_{N_{1}^{(k)}}>s_{N_{1}^{(k)}-1}$ we infer that the "density" of $A$ will not be affected in the interval $\left[s_{N_{3}^{(k-1)}}, s_{N_{3}^{(k)}}\right)$ if we select further elements $a_{N_{3}^{(k)}+1}, a_{N_{3}^{(k)}+2}, \ldots$

For $x=a_{k_{0}}$ by the definition of $a_{k_{0}}$ we get

$$
\alpha \leq p\left(a_{k_{0}}\right) / a_{k_{0}} \leq \beta \text {. }
$$

Now let $k>k_{0}$ and assume that $s_{N_{3}^{(k-1)}} \leq x \leq s_{N_{3}^{(k)}}$. We use the abbreviations $N_{i}=N_{i}^{(k)}, i=1,2,3$, and $N_{0}=N_{3}^{(k-1)}$ again.

1. Let $s_{N_{0}} \leq x<a_{N_{1}}$. Since $p_{N_{0}}(x) / x$ is a decreasing function of $x$ in this interval we have, by (6),

$$
\alpha \leq p\left(a_{N_{1}}\right) / a_{N_{1}} \leq p(x) / x \leq p_{N_{0}}\left(s_{N_{0}}\right) / s_{N_{0}} \leq \beta .
$$

2. Let $a_{N_{1}} \leq x \leq s_{N_{2}}$ and let $j a_{N_{1}} \leq x<(j+1) a_{N_{1}}$ for some $1 \leq j \leq$ $\left(\begin{array}{c}M+t+1 \\ 2\end{array}\right)$. Let $x^{\prime}=x-j a_{N_{1}}$. By the definition of $a_{N_{1}+1}, \ldots, a_{N_{2}}$ we conclude by (9) and (11) that

$$
p_{N_{2}}(x) / x=\left\{j\left|P\left(A_{N_{1}}\right)\right|+\varepsilon\left|P\left(A_{N_{0}}\right)\right|+p_{N_{2}}\left(x^{\prime}\right)\right\} / x,
$$

where $\varepsilon=0$ if $1 \leq j \leq\left(\begin{array}{c}M+1 \\ 2\end{array}\right)$ and $\varepsilon=t-1$ if $\left(\begin{array}{c}M+1 \\ 2\end{array}\right) \leq j \leq\left(\begin{array}{c}M+t+1 \\ 2\end{array}\right)$ i.e. if $\left.t=\left[a_{N_{1}} / s_{N_{0}}\right] \geq 2\right)$. The inductive hypothesis

$$
a_{N_{1}} \alpha \leq\left|P\left(A_{N_{1}}\right)\right| \leq a_{N_{1}} \beta, \quad \alpha s_{N_{0}} \leq p\left(s_{N_{0}}\right) \leq \beta s_{N_{0}},
$$

and $\alpha x^{\prime} \leq p_{N_{2}}\left(x^{\prime}\right) \leq \beta x^{\prime}$ yield

$$
p_{N_{2}}(x) / x \leq\left\{j t s_{N_{0}} \beta+\beta x^{\prime}\right\} / x \leq \beta\left\{j\left(a_{N_{1}} / s_{N_{0}}\right) s_{N_{0}}+x^{\prime}\right\} / x=\beta
$$


and

$$
p_{N_{2}}(x) / x \geq\left\{j a_{N_{1}} \alpha+\alpha x^{\prime}\right\} / x=\alpha\left\{j a_{N_{1}}+x^{\prime}\right\} / x=\alpha .
$$

3. $s_{N_{2}}<x \leq s_{N}$ where $N=N_{2}+v$ was defined in (14). By (14),

$$
\left[a_{N_{2}+i}, a_{N_{2}+i+1}\right] \cap P\left(A_{N}\right)=a_{N_{2}+i}+P\left(A_{N_{2}}\right) \text {. }
$$

Thus if $a_{N_{2}+i} \leq x<2 a_{N_{2}+i}$ and $x^{\prime}=x-a_{N_{2}+i}$ then by the inductive hypothesis again and by (24),

$$
p_{N}(x) / x \leq\left\{\beta a_{N_{2}+i}+x^{\prime} \beta\right\} / x=\beta
$$

and

$$
p_{N}(x) / x \geq\left\{\alpha a_{N_{2}+i}+x^{\prime} \alpha\right\} / x=\alpha .
$$

4. Finally, let $x \in\left[a_{N+1}, s_{N+1}\right]$. Since $a_{N+1}<s_{N}$ it follows that if $x \leq s_{N}$ then $p_{N}(x) / x \geq \alpha$. This implies that

$$
p_{N+1}(x) / x \geq \alpha
$$

for every $x \in\left[a_{N+1}, s_{N+1}\right]$.

Now we only have to prove that $p_{N+1}(x) / x \leq \beta$. If $x \geq W$ then by $Y \leq W\left(\beta / \varrho_{N}-1\right)$ we have

$$
\begin{aligned}
p_{N+1}(x) / x & \leq\left\{\varrho_{N} x+Y \varrho_{N}\right\} / x=\varrho_{N}+Y \varrho_{N} / x \\
& \leq \varrho_{N}+Y \varrho_{N} / W \leq \varrho_{N}+\left(\beta-\varrho_{N}\right)=\beta .
\end{aligned}
$$

If $a_{N+1} \leq x<W$ then

$$
p_{N+1}(x) / x=\varrho_{N} x+\left(x-a_{N+1}\right) \varrho_{N} / x<\varrho_{N}+Y \varrho_{N} / W=\beta .
$$

Now, to define $a_{N+2}, a_{N+3}, \ldots$ by (14) and (19) we can apply the same ideas as in items 3 and 4 ; in this way we conclude that (25)-(28) hold for every $x$ with $s_{N} \leq x \leq s_{N_{3}}$, so that (5) holds and this completes the proof of the Theorem.

\section{References}

[1] P. Erdős and E. Szemerédi, On sums and products of integer, in: Studies in Pure Mathematics, To the Memory of P. Turán, Akadémiai Kiadó, 1983, 213-218.

[2] N. Hegyvári, On two problems in the combinatorial number theory (in Hungarian), to appear.

[3] I. Z. Ruzsa, The density of the set of sums, Acta Arith. 58 (1991), 169-172.

DEPARTMENT OF MATHEMATICS, ELTE TFK

L. EÖTVÖS UNIVERSITY

MARKÓ U. 29

1055 BUDAPEST, HUNGARY

Received on 15.6.1993

and in revised form on 28.2.1994 